\title{
Clustering FunFams using sequence embeddings improves EC purity
}

\author{
Maria Littmann $(\mathbb{1}){ }^{1,2}, *$, Nicola Bordin $\mathbb{1}^{3}{ }^{3}$, Michael Heinzinger ${ }^{1,2}$, Konstantin Schütze ${ }^{1}$, \\ Christian Dallago (1) ${ }^{1,2}$, Christine Orengo ${ }^{3, *, \dagger}$ and Burkhard Rost ${ }^{1,4,5, \dagger}$
}

1Department of Informatics, Bioinformatics \& Computational Biology-i12, TUM (Technical University of Munich), 85748 Garching/ Munich, Germany, ${ }^{2}$ Center for Doctoral Studies in Informatics and its Applications (CeDoSIA), TUM Graduate School, Center of Doctoral Studies in Informatics and its Applications (CeDoSIA), 85748 Garching/Munich, Germany, ${ }^{3}$ Institute of Structural and Molecular Biology, University College London, London WC1E 6BT, UK, ${ }^{4}$ Institute for Advanced Study (TUM-IAS), 85748 Garching/ Munich, Germany and ${ }^{5}$ TUM School of Life Sciences Weihenstephan (WZW), 85354 Freising, Germany

*To whom correspondence should be addressed.

${ }^{\dagger}$ The authors wish it to be known that, in their opinion, the last two authors should be regarded as Joint Last Authors.

Associate Editor: Pier Luigi Martelli

Received on January 25, 2021; revised on April 2, 2021; editorial decision on May 10, 2021; accepted on May 11, 2021

\begin{abstract}
Motivation: Classifying proteins into functional families can improve our understanding of protein function and can allow transferring annotations within one family. For this, functional families need to be 'pure', i.e., contain only proteins with identical function. Functional Families (FunFams) cluster proteins within CATH superfamilies into such groups of proteins sharing function. 11\% of all FunFams (22 830 of 203639 ) contain EC annotations and of those, $7 \%$ (1526 of 22 830) have inconsistent functional annotations.

Results: We propose an approach to further cluster FunFams into functionally more consistent sub-families by encoding their sequences through embeddings. These embeddings originate from language models transferring knowledge gained from predicting missing amino acids in a sequence (ProtBERT) and have been further optimized to distinguish between proteins belonging to the same or a different CATH superfamily (PB-Tucker). Using distances between embeddings and DBSCAN to cluster FunFams and identify outliers, doubled the number of pure clusters per FunFam compared to random clustering. Our approach was not limited to FunFams but also succeeded on families created using sequence similarity alone. Complementing EC annotations, we observed similar results for binding annotations. Thus, we expect an increased purity also for other aspects of function. Our results can help generating FunFams; the resulting clusters with improved functional consistency allow more reliable inference of annotations. We expect this approach to succeed equally for any other grouping of proteins by their phenotypes.
\end{abstract}

Availability and implementation: Code and embeddings are available via GitHub: https://github.com/Rostlab/ FunFamsClustering.

Contact: littmann@rostlab.org or c.orengo@ucl.ac.uk or assistant@rostlab.org

Supplementary information: Supplementary data are available at Bioinformatics online.

\section{Introduction}

Knowledge about the function of a protein is crucial for a wide array of biomedical applications. Classifying protein sequences into functional families can shed light on uncharacterized proteins. Functional families can also reveal insights into the evolution of function through sequence changes (Hannenhalli and Russell, 2000). To gain meaningful insights, those families should be consistent, i.e., only contain functionally similar proteins.
CATH FunFams (Orengo et al., 1997; Sillitoe et al., 2020) provide a functional sub-classification of CATH superfamilies (Das et al., 2016; Sillitoe et al., 2013). Superfamilies are the last level $(\mathrm{H})$ in the CATH hierarchy; they group sequences which are related by evolution, often loosely referred to as homologous. While proteins in one superfamily can still be functionally and structurally diverse, Functional Families (FunFams) further sub-classify superfamilies into coherent subsets of proteins with the same function. FunFams can be used to predict function on 
a per-protein level as described through Gene Ontology (GO) terms (Das et al., 2015; Zhou et al., 2019), to predict functional sites (Das et al., 2020), or to improve binding residue predictions through consensus (Scheibenreif et al., 2019).

The Enzyme Commission number (EC number) (Webb, 1992) numerically classifies enzymatic functions based on the reactions they catalyze. It consists of four levels, and each level provides a more specific description of function than the previous one. The function of two proteins is more similar, the more levels of their two EC numbers are identical, particularly, for the levels EC3 and EC4, which describe the chemical reaction and its substrate specificity.

For 22830 FunFams (11\% of all), manually curated annotations from UniProt (The UniProt Consortium, 2019) for EC numbers for all four levels are available at least for one member. By design, proteins from the same FunFam should share the same EC class (annotated up to level 4). However, 1526 FunFams (7\% of 22830 ) accounting for $16 \%$ of all sequences in the 22830 FunFams with EC annotations have more than one annotation, and 180 (1\% of 22 830 ) accounting for $2 \%$ of the sequences even have four or more different annotations (Supplementary Fig. S1). Different EC annotations within one FunFam could originate from multifunctional enzymes [e.g., promiscuous enzymes (Khersonsky and Tawfik, 2010) or moonlighting enzymes (Jeffery, 2003)]. Assuming the multifunctional enzyme to have two EC numbers, only one of those could be inconsistent with the other FunFam members rendering that FunFam seemingly inconsistent. However, different EC annotations can also result from impurity, i.e., FunFams containing proteins with different functions. Splitting FunFams further could provide a more fine-grained and consistent set of functionally related proteins.

Over the last few years, novel representations (embeddings) for proteins have emerged from adapting language models (LMs) developed for natural language processing (NLP) to protein sequences (Alley et al., 2019; Elnaggar et al., 2020; Heinzinger et al., 2019; Madani et al., 2020; Rao et al., 2019). These embeddings are learnt solely from protein sequences either through auto-regressive pretraining [predicting the next amino acid in a sequence, e.g., ELMo (Peters et al., 2018) or GPT (Radford et al., 2018)] or through masked language modeling [reconstructing corrupted amino acids from the sequence, e.g., BERT (Devlin et al., 2019)] without using any annotations (self-supervised) or any knowledge of evolutionary constraints. To accomplish this, the protein LM learns some aspects of the language of life as written in protein sequences (Heinzinger et al., 2019). Features learnt implicitly by these models can be transferred to any task by extracting the hidden states of the LM for a given protein sequence (transfer learning). These hidden states are referred to as the embeddings of the corresponding LM. Such embeddings capture higher-level features of proteins, including various aspects of protein function (Asgari and Mofrad, 2015; Hamid and Friedberg, 2019; Littmann et al., 2021; Rives et al., 2020; Vig et al., 2020; Villegas-Morcillo et al., 2020; Yang et al., 2018). Therefore, we hypothesized that this orthogonal perspective-using embedding rather than sequence space-might help to find functionally consistent sub-groups within protein families built using sequence similarity.

Here, we proposed a clustering approach to identify clusters in FunFams that are more consistent in terms of shared functionality. To this end, shared functionality was defined as sharing the same EC annotation up to the fourth level (i.e., completely identical EC numbers). We represented protein sequences as embeddings, i.e., fixed-size vectors derived from pre-trained LMs.

We used the LM ProtBERT (Elnaggar et al., 2020) to retrieve the initial embeddings, and applied contrastive learning (Becker and Hinton, 1992; Bromley et al., 1993; Le-Khac et al., 2020) using the triplet loss (Weinberger and Saul, 2009) to learn a new embedding space which was optimized toward maximizing the Euclidean distance between proteins from different CATH classes while minimizing the distance between proteins in the same CATH class. During training, the distance in embedding space between similar proteins is decreased and between dissimilar proteins is increased. Similarity was defined based on the CATH hierarchy.
The resulting embeddings are called PB-Tucker. Clustering was then performed based on the Euclidean distances between those embeddings using DBSCAN (Ester et al., 1996). Within each FunFam, DBSCAN identified clusters as dense regions in which all sequences were close to each other in embedding space; it classified proteins as outliers if they were not close to other sequences in the FunFam. That allowed the identification of (i) a more fine-grained clustering of the FunFams, and (ii) single sequences which might have been falsely assigned to this FunFam. Analyzing whether or not embedding-based clustering reduced the number of different EC annotations in a FunFam allowed validating our new approach.

\section{Materials and methods}

\subsection{FunFams dataset}

The current version of CATH (v4.3) holds 4328 superfamilies split into 212872 FunFams. The FunFams generation process, albeit changing through time, consists of various steps, starting with the clustering of all sequences within a CATH superfamily at $90 \%$ sequence similarity, encoding these clusters in Hidden Markov Models and creating a relationship tree between all clusters using GeMMA (Lee et al., 2010) and HHsuite (Steinegger et al., 2019). Subsequently, CATH-FunFHMMer (Das et al., 2015) is applied to traverse the tree, and GroupSim (Capra and Singh, 2008) conservation patterns are employed to merge or cut the tree branches to obtain the largest possible, functionally pure family. CATH FunFams have higher functional purity than CATH superfamilies, and conserved residues are enriched in functional sites (Das et al., 2015).

\subsection{EC annotations and EC purity}

EC annotations for the FunFams dataset were obtained using the UniProt (The UniProt Consortium, 2019) SPARQL API and crossassigned to all UniProt IDs available within the FunFams. UniProt provides manually curated annotations combining multiple sources through UniRule (MacDougall et al., 2020) and using the standardized vocabulary from Rhea (Morgat et al., 2020).

Since proteins in the same FunFam are assumed to share a function, we expect all proteins in one FunFam to have the same EC number(s). If not, the FunFam is considered impure, i.e., it contains sequences which belong in another FunFam. If all proteins were annotated with a single EC number, impurity could naively be defined as any FunFam with more than one unique EC number (i.e., at least one protein is annotated to another EC than the other family members). However, some proteins are annotated with multiple EC numbers. These proteins might either execute multiple functions (Jeffery, 2003; Khersonsky and Tawfik, 2010) or might be mis-classified. In fact, $8 \%$ (7 586) of all proteins with EC annotations in our final dataset are annotated to more than one EC (Supplementary Table S1).

Consider the following two FunFams: FF1 has several proteins all annotated with the same two EC numbers EC1 and EC2, while FF2 has one protein with EC1 another with EC2. Then, we consider FF1 as pure and FF2 as impure. The purity of clusters was defined accordingly. We only considered EC annotations with all four levels; all others were considered un-annotated.

\subsection{Embeddings representing proteins (PB-Tucker)}

We used ProtBERT (Elnaggar et al., 2020) to create fixed-length vector representations (embeddings), i.e., vectors with the same number of dimensions irrespective of protein length. ProtBERT uses the architecture of the LM BERT (Devlin et al., 2019) which applies a stack of self-attention (Bahdanau et al., 2016) layers for masked language modeling (details in Supporting Online Material Section SOM_1.2). Fixed-length vectors were derived by averaging over the representations of each amino acid extracted from its last layer. This simple global average pooling provides an effective baseline (Elnaggar et al., 2020; Heinzinger et al., 2019; Rives et al., 2020). In the following, ProtBERT refers to this representation. 
We applied contrastive learning (Becker and Hinton, 1992; Bromley et al., 1993; Le-Khac et al., 2020) on the ProtBERT embeddings to learn a new embedding space, which was optimized to increase the distance between CATH superfamilies and brings those within one superfamily closer, while pushing members of different superfamilies apart. Toward this end, we used the triplet loss (Weinberger and Saul, 2009) to optimize Euclidean distances between protein triplets, i.e., an anchor protein is compared to a positive and a negative protein. During training, the distance in embedding space between anchor and positive is decreased, that between anchor and negative is increased. The notion of positive and negative was taken from the CATH hierarchy.

While supervised learning for a CATH-like hierarchy is challenging, using a hierarchy to define relative similarity between triplets is straightforward as anchor and positive only need to share one level more in the hierarchy than anchor and negative. Toward this end, ProtBERT representations were projected in two steps from 1024dimensions (1024-d) to 128-d using CATH v4.3 (Sillitoe et al., 2020) for training the two-layer neural network (SOM_1.2). In the following, we call these new 128-d embeddings PB-Tucker (Heinzinger et al., unpublished). PB-Tucker has been trained to differentiate CATH superfamilies and seemed to better capture functional relationships between proteins in one superfamily than the original ProtBERT (SOM_1.2).

\subsection{Clustering}

Representing sequences as PB-Tucker embeddings, we calculated the Euclidean distance between all embeddings within one FunFam. The distance $d$ between two embeddings $x$ and $y$ was defined as:

$$
d(x, y)=\sqrt{\sum_{i=1}^{128}\left(x_{i}-y_{i}\right)^{2}}
$$

Alternatively, we tested Cosine distance (Equation 2), commonly used for embeddings, and Manhattan distance (Equation 3), commonly used for high-dimensional data.

$$
\begin{gathered}
d_{\text {cosine }}(x, y)=\frac{\sum_{i=1}^{128} x_{i} y_{i}}{\sqrt{\sum_{i=1}^{128} x_{i}^{2} \cdot \sqrt{\sum_{i=1}^{128} y_{i}^{2}}}} \\
d_{\text {manhattan }}(x, y)=\sum_{i=1}^{128}\left|x_{i}-y_{i}\right|
\end{gathered}
$$

Based on the Euclidean distances, we clustered all sequences within one FunFam using the implementation of DBSCAN (Ester et al., 1996) in scikit-learn (Pedregosa et al., 2011). For a set of data points, DBSCAN identifies dense regions, i.e., regions of points close to each other, and classifies these regions as clusters. Data points not close to enough other data points are classified as outliers. DBSCAN identifies core points to seed a cluster; all points within a certain distance of the core point are added to this cluster. The two free parameters are: (i) the distance cutoff $\theta$ to consider points close (points $\mathrm{A}$ and $\mathrm{B}$ are considered close, if $\mathrm{d}(\mathrm{A}, \mathrm{B})<\theta$ ), and (ii) the number of neighbors $n$ (including the point itself) required for a point to become 'core point'; $n$ implicitly controls the size and number of clusters. For our application, DBSCAN has two major advantages: (i) the number of clusters does not have to be set a priori, and (ii) clustering and outlier detection are simultaneous.

If not stated otherwise, we used the default $n=5$ although it has been suggested to use values between $n=\mathrm{D}+1$ and $n=2 * \mathrm{D}-1$, where $\mathrm{D}$ is the number of dimensions (Sander et al., 1998). With $\mathrm{d}=128$ for the PB-Tucker embeddings, that implies $n=255$. Since FunFams vary in size, $n$ might be adjusted to that size. For five superfamilies, we tested, in addition to $n=5, n=129, n=255$ as fixed neighborhood sizes, as well as $n=0.05^{*}|\mathrm{~F}|, n=0.1^{*}|\mathrm{~F}|$, $n=0.2^{*}|\mathrm{~F}|(|\mathrm{F}|=$ number of sequences in FunFam $)$ as variable neighborhood sizes dependent of the size of the FunFam.

Observing differences in the distances between the members of different superfamilies (Supplementary Fig. S3), it appeared best to choose superfamily-specific values for $\theta$. Initially, we considered using expected distance between any two members of the same FunFam. However, large distances between members in one FunFam might reveal impurity rather than a generic width of a family. Instead, we computed the median over those distances for all FunFams in one superfamily and used this value for each FunFam. The resulting value still reflects the expected distance between pairs, but the effect of large distances due to impurity should be averaged out by considering all FunFams in a superfamily. In detail, for each member in each FunFam, we calculated its average distance to all other members of that FunFam (distance distribution for five superfamilies in Supplementary Fig. S3). Given the distribution of these average sequence distances, we chose the median distance as $\theta$, i.e., we chose a distance cutoff so that $50 \%$ of all sequences in a superfamily were on average within a distance of $\theta$ to all other sequences in the same FunFam. Decreasing $\theta$ raises outliers and yields smaller clusters, while increasing $\theta$ reduces outliers and yields larger clusters.

\subsection{Measuring purity of clustered FunFams}

To estimate whether the clustering of an impure FunFam led to more consistent sub-families, we calculated the percentage of pure clusters. Clusters with no EC annotation were excluded. For each FunFam, we calculated the clusters with one single EC annotation as percentage of all clusters with EC annotations and defined this measure as the purity of a FunFam (Equation 4). We then defined the percentage of completely pure FunFams as the percentage of FunFams with a purity of 100 .

$$
\operatorname{Purity}(F)=\frac{\text { clusters }_{\text {pure }}}{\text { clusters }_{\text {withECs }}} \cdot 100
$$

We also calculated the purity of a FunFam in terms of its size, i.e., the number of sequences contained in it:

$$
\text { Purity }_{\text {Sequences }}(F)=\frac{\text { sequencesinpureclusters }}{\text { sequencesinclusterswithECs }} \cdot 100
$$

\subsection{Confidence intervals (Cls)}

$95 \%$ symmetric confidence intervals (CIs) were calculated from 1 , 000 bootstrap samples to indicate the spread of data and certainty of average values.

\subsection{Final dataset}

To construct the dataset used in this analysis, we extracted all superfamilies with at least one impure FunFam, i.e., at least one FunFam with more than one EC annotation. Since embeddings could only be computed for continuous sequences, we excluded sequences with multiple segments. After this removal, some FunFams became orphans (consisting of only a single sequence) and were also excluded. This led to a final dataset of 458 superfamilies $(10.6 \%$ of all superfamilies) with 110876 FunFams (52.1\%) and 13011 $(6.1 \%)$ with EC annotations. Those 13011 FunFams accounted for $20 \%$ of all proteins in the FunFams (1 669245 sequences). All FunFams in a superfamily were used to determine the clustering distance cut-off $\theta$. However, only FunFams with EC annotations were clustered to save computer time, and hence, energy.

\subsection{Availability}

The final dataset and the corresponding embeddings as well as the source code used for clustering are publicly available via GitHub: https://github. com/Rostlab/FunFamsClustering. In addition, ProtBERT and PB-Tucker embeddings for any sequence can be retrieved using the bio_embeddings pipeline (Dallago et al., 2021). 


\section{Results}

\subsection{Embedding clusters increased EC purity}

We began with 13011 FunFams (6\% of all) with at least one EC annotation. Of these, $1273(10 \%)$ contained more than one EC annotation (impure FunFams). Applying DBSCAN to all EC annotated FunFams, we split the 13011 into 26464 clusters (21 546 for pure and 4918 for impure FunFams). On average, 4.4-4.6\% (95\% confidence interval, CI) of the proteins in a FunFam were classified as outliers (Supplementary Table S4). 63\% of the DBSCAN clusters contained proteins with EC annotations; only $4 \%$ of those contained multiple EC annotations (versus $10 \%$ in all FunFams; Fig. 1A). Only $10 \%$ of all proteins (155 044 of 1593 567) belonged to clusters with multiple EC annotations compared to $21 \%$ (356 565of 1668 273) for FunFams (Fig. 1B). Thus, a larger fraction of clusters was pure (i.e., contained one EC annotation) than of FunFams both in terms of numbers of clusters and numbers of proteins (Fig. 1).

To further understand the extent to which the clustered FunFams provide a functionally more consistent subset, we determined for each impure FunFam, the fraction of clusters that were pure (Methods). To begin: 37\% of all clusters had no EC annotated proteins and were excluded from further analysis. Of the remaining 16906 clusters (63\%), 22\% were impure, i.e., contained more than one EC annotation. On average, $63 \%$ (CI [60-66\%]) of the clusters for a FunFam were pure (Fig. 2; dashed blue line) accounting for $58 \%$ (CI [55-61\%]) of all proteins (Fig. 2; dotted red line). 52\% of all impure FunFams were split into completely pure clusters, i.e., for every other FunFam, the embedding-split clustered into functionally consistent sub-families (Fig. 2, right most blue point ' $100 \%$ Pure
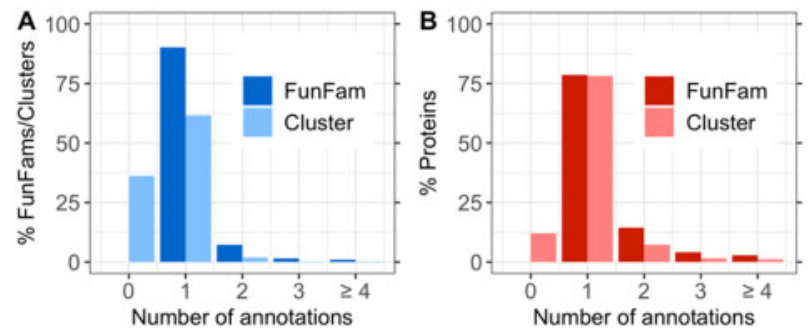

Fig. 1. EC purity for FunFams and embedding clusters. This analysis considered 13 011 FunFams with EC annotations. (A) The distribution of all families (FunFam/ Clusters), i.e., the percentage of FunFams and embedding-based clusters with $n$ EC annotations ( $n \geq 1$ for FunFams and $n \geq 0$ for new clusters; note: bars left and right of integer values $n$, not separated by a white space denote $n$ annotations). (B) The distribution of all proteins, i.e., the percentage of proteins in families (FunFam/ Cluster) with $n$ EC annotations. This number does not reveal how many proteins have an EC annotation. Of the 13011 FunFams, $10 \%$ were impure, i.e., had multiple EC annotations (100-value for dark blue bar at 1 in panel A), and $21 \%$ of all proteins were part of these impure FunFams. After embedding-based split of FunFams, 64\% (16 906) of the resulting clusters contain ECs (100-light blue bar at 0 ) and 4\% (606) of those 16906 were annotated to more than one EC accounting for $11 \%$ of proteins in clusters with ECs.

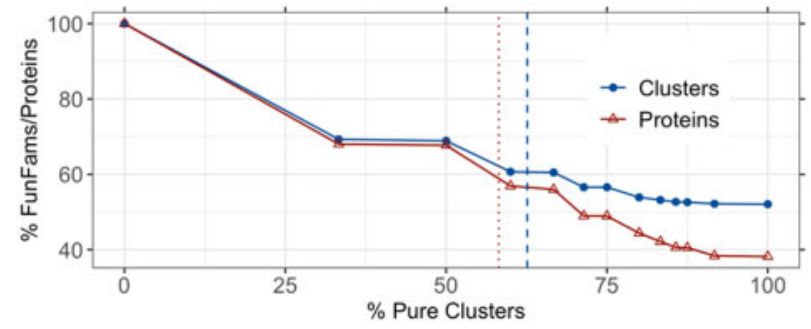

Fig. 2. Fraction of pure clusters for impure FunFams. The y-axis gives the percentages of all clusters (blue line) or of all proteins (red line) in FunFams at levels of increasing cluster purity (Equations 4,5 ). On average, $63 \%$ of the clusters for a FunFam were pure (dashed vertical blue line) accounting for $58 \%$ of the proteins (dotted vertical red line). $52 \%$ of the impure FunFams were split only into pure clusters (right most blue point) accounting for $38 \%$ of the proteins.
Clusters') accounting for 38\% of all proteins (Fig. 2, right most red point). This measure gave conservative estimates as it only considered completely pure clusters, ignoring improvements through reduction of EC annotations, e.g., when a group had originally $m+1$ annotations and the clustering improved to $m$, this improvement was ignored for all $m>1$.

\subsection{Improving EC purity without over-splitting}

While splitting impure FunFams through embedding-based clustering clearly improved the EC purity, we wanted to avoid over-splitting. Trivially, the more and smaller clusters, the more likely they are pure. In the non-sense extreme of $\mathrm{N}$ clusters for $\mathrm{N}$ sequences, all clusters are trivially pure. One constraint to avoid generating too many clusters (over-splitting) is to do substantially better than by randomly splitting into the same number of clusters. We computed the random clustering using the same cluster sizes and outlier numbers as realized by the embedding-based clustering. Fewer than half as many embedding-based clusters were impure than for random (Fig. 3A, $47 \pm 1 \%$ versus $22 \pm 1 \%$ ). Similarly, the average purity of a FunFam was almost double for embedding than for random clustering (Fig. 3B, $63 \pm 3 \%$ versus $38 \pm 5 \%$ ), and 3.5 times more FunFams were split into exclusively pure clusters by the embeddingbased clustering (Fig. 3C, $52 \pm 3 \%$ versus $15 \pm 1 \%$ ). This corresponded to $4.8 \%$ (CI [4.4-5.2\%]) of all proteins clustered into pure clusters at random compared to $38 \%$ (CI [33-43\%]) of all proteins for embedding-based clustering, i.e., an over 7-fold increase (Fig. $3 \mathrm{C}$, red bars).

An ideal split of impure FunFams generates clusters defined by two features: all members share the same EC annotation(s) and all proteins with the same EC annotation(s) are in the same cluster. Ignoring the latter leads to over-splitting. For the embedding-based clustering, $81 \%$ of the ECs occurred in one cluster (Fig. 4). However, some of the outliers had EC annotations. When also counting those (as single member clusters), the percentage of EC-exclusive clusters dropped to $63 \%$ (Fig. 4). Thus, the embedding-based clustering largely avoided over-splitting. Nevertheless, $8 \%$ of all experimentally known EC numbers were annotated to proteins from at least three different clusters (17\% if including outliers; Fig. 4) and some $(10 \%)$ of the outliers shared the EC number with the cluster from which they had been removed. This might indicate over-splitting or suggest a more fine-grained functional distinction between those proteins than is captured in the fourth EC level.

If the increased purity through clustering had been a random effect, the embedding space clustering would be EC-independent. If so, we expect no difference in the distributions of embedding distances between pure and impure FunFams, and a similar number of clusters and outliers. However, pure FunFams were, on average,
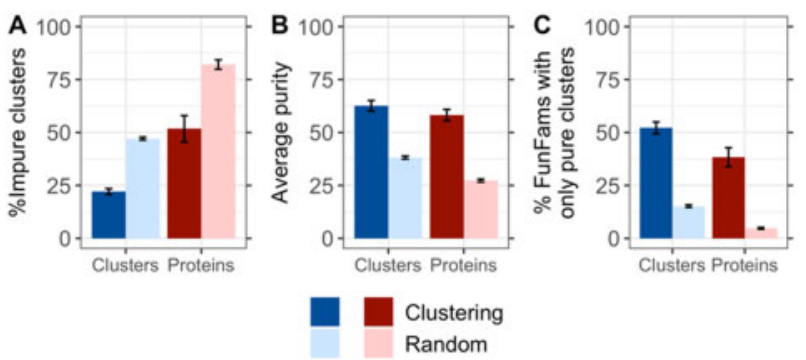

Fig. 3. Embedding-based clusters improve EC purity over random. Random clusters were computed using the same cluster size and outlier number realized by the embedding-based clustering, but the FunFam members were randomly assigned. (A) The fraction of impure clusters was higher for the random clustering than for our clustering (29\% versus 12\%). (B) Through DBSCAN embedding-based clustering, each impure FunFam was, on average, split into $63 \%$ pure clusters while for the random clustering, the average purity was only $38 \%$. (C) More than half of all FunFams $(53 \%)$ were split only into pure clusters for embedding-based clustering but only $15 \%$ for a random clustering. Error bars indicate symmetric $95 \%$ confidence intervals. Blue colored bars indicate numbers in terms of clusters, red colored bars in terms of proteins in those clusters. Darker colors indicate values for the clustering while lighter colors indicate values for the random approach. 


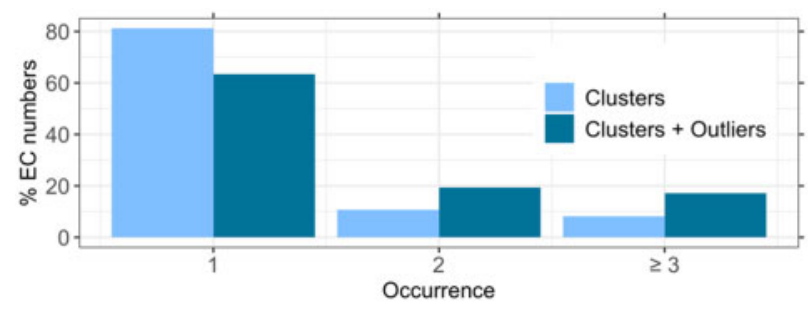

Fig. 4. Most EC numbers only occur in one cluster. For each EC number in a FunFam, we counted the number of embedding-based clusters in which it occurred to gauge potential over-splitting. $81 \%$ of the ECs only occurred in one cluster (darker bars). If we considered outliers as clusters with one member, this number dropped to $63 \%$. These results suggest that the clustering did not over-split the FunFams and that functionally related proteins ended up in the same cluster.

split into only two clusters, while impure FunFams were split into four clusters (Supplementary Table S4). This finding suggested that if a FunFam is split into many clusters, it should be considered for further manual inspection to establish whether all proteins were correctly assigned to this functional family (SOM_2.1).

\subsection{Different levels of EC annotations gave similar results}

Up to this point, we only distinguished whether two proteins had the same EC annotation or not, ignoring that two proteins with ECs A.B.C.X and A.B.C.Y more likely have similar molecular function than a pair with A.* and D.*. Pairs of the first type (difference only in 4th level) will, on average, be more sequence similar than pairs of the second type (difference in 1st level). Most impure FunFams were impure due to differences on the fourth level of EC annotations (Supplementary Fig. S5). Although we analyzed the clustering at higher levels of the EC classification, the results were inconclusive, probably due to data sparsity (SOM_2.2). The results for five specifically chosen superfamilies were similar (Supplementary Fig. S7) underlining the more general findings that the level of EC annotation causing impurity did not crucially affect the embedding-based clustering (SOM_3.2). Instead, the performance was likely impacted more by other factors such as the presence of multifunctional enzymes and by missing annotations, i.e., an insufficient coverage by proteins with explicit experimental evidence. We applied a rather conservative definition of purity: If one protein were annotated to two EC numbers $(\mathrm{EC} 1+\mathrm{EC} 2)$ and another protein in the same cluster were only annotated to one of those two (EC1), we would consider this cluster impure. If this difference were not due to missing annotations, we could group the two into two separate families (those with EC1+EC2, those with only EC1).

Although considering those FunFams or clusters as impure is too strict, not doing this is too loose because it will raise the performance of the random approach given that many families have many EC numbers. More importantly, the EC1-only proteins may have annotations that are incomplete or incorrect.

\subsection{Slightly worse results for experimentally verified annotations}

Not all EC annotations in UniProt are experimentally verified. Only using experimentally verified EC annotations (with evidence code ECO: 0000269) for sequences in Swiss-Prot, 4709 FunFams contained any sequence with EC annotations and $637(14 \%)$ were impure.

$46 \%$ of those impure FunFams were clustered into fully pure clusters, and our approach achieved an average purity of $53 \%$ being slightly worse than for the full set. This could be due to missing annotations: If two proteins A and B in a family or cluster are annotated to the same two EC numbers EC1 and EC2, but for protein A only EC1 is experimentally verified and for protein B EC2, such a family or cluster would be considered impure. This explanation is also supported by the fact that a larger fraction of FunFams was impure if only experimentally verified annotations were considered (14\% versus $10 \%)$.
3.5 Similar improvement for single domain proteins

Most EC annotations are available for an entire protein, not for the structural domain responsible for this function, yet most proteins have several domains (Liu and Rost, 2004). To avoid the potential problem of multi-domain proteins for purity, we assessed the clustering results for 2412 FunFams only consisting of single-domain proteins. Of those, $6 \%$ (136) were impure versus $10 \%$ for all FunFams. This provided a rough idea for the problem of our purity definition: roughly half $(10 / 6 \sim 2)$ of the problem related to multi-domain proteins.

Clustering single domains performed similarly to full-length proteins: $52 \%$ of the impure FunFams with single-domain proteins were clustered into fully pure clusters versus $52 \%$ for the entire set, and the clustering achieved an average purity of $60 \%$ (versus $63 \%$ ). These results showed that the clustering approach did not only remove the impurity caused by inaccurate annotations but could identify functional relationships not detected using sequence comparison.

\subsection{Details of parameter choices mattered}

For a more detailed analysis of particular details of our method, in particular, for the choice of embeddings and clustering parameters, we chose five superfamilies with diverse properties (CATH identifiers: 3.40.50.150, 3.20.20.70, 3.40.47.10, 3.50.50.60, 1.10.630.10; SOM_3).

Embedding space better captured functional sub-groups than sequence space

Instead of using embedding distance for DBSCAN, we redid the clustering using the pairwise percentage sequence identity (PIDE) between any two sequences in a FunFam, converted into a sequence distance (1-PIDE). This approach generated more clusters with more outliers that were, on average, less pure than from embeddings (Supplementary Table S7). This implied that embedding similarity captured functional relationships between sequences better than sequence similarity.

Euclidean distance yielding best clustering results

Cosine distance is often considered more standard for comparing embeddings, e.g., in NLP. Replacing Euclidean distance with Cosine distance (Equation 2) revealed that most embeddings of proteins within a FunFam were represented by vectors with the same orientation leading to a Cosine distance of 0 , i.e., we could not use it to split any FunFam (Supplementary Table S7). Euclidean distance often suffers more from problems with high dimensional spaces (Beyer et al., 1999) than Manhattan distance (Equation 3). Indeed, for our problem Manhattan, unlike cosine, distance worked but not better than Euclidean (Supplementary Table S7). Since PB-Tucker was optimized on Euclidean distance, we clustered on Euclidean.

PB-Tucker embeddings largely superior to ProtBERT embeddings In direct comparison between ProtBERT and PB-Tucker embeddings, we observed the following when clustering the five superfamilies (Supplementary Table S3): The number of clusters and outliers was slightly smaller for ProtBERT, but the fraction of impure clusters was higher than for PB-Tucker (19\% for ProtBERT versus $13 \%$ for 'default'; Supplementary Table S7). The average purity was also higher for PB-Tucker ('default' $=59 \%)$ than for ProtBERT $(51 \%$; Supplementary Table S7). Thus, PB-Tucker appeared superior in capturing functional differences.

Smaller distance thresholds resulted in smaller and purer clusters The distance threshold $\theta$ of DBSCAN defines whether or not two points are close enough to each other to be grouped. For the default clustering, we chose the median distance between all proteins for each superfamily (Methods). The observed distribution of distances (Supplementary Fig. S3) suggested choosing superfamily-specific thresholds. As expected, the smaller $\theta$, the more clusters and outliers will result (' $\theta=1$ st quartile' versus 'default'; Supplementary Table S7). Largely due to splitting FunFams into more clusters at smaller $\theta$, the resulting clusters were seemingly purer with only $4 \%$ impure 
clusters (versus $13 \%$ ) and an average purity of $83 \%$ (versus $59 \%$; Supplementary Table S7). In contrast, larger $\theta$ thresholds (here the 3rd quartile) affected fewer, more impure clusters (Supplementary Table S7). Thus, the choice of $\theta$ highly influenced the clustering results. For some applications, lower values of $\theta$ might be best to obtain a large, highly consistent set of small sub-families that can serve, e.g., as seed to further extend those sub-families to larger functionally related families. Also, especially for FunFams for which using a larger cutoff largely failed, decreasing the distance threshold can help to still identify which sequences might cause impurity.

Default neighborhood size resulted in best clustering

DBSCAN forms clusters around 'core points' which are points with at least $n$ neighbors. For the five superfamilies, we tested fixed neighborhood sizes of $n \varepsilon[5 ; 129 ; 255]$ and variable neighborhood sizes dependent on the size of the FunFam $n=x^{*}|\mathrm{~F}|$, with $|\mathrm{F}|$ as the number of proteins in a FunFam and $x \varepsilon[0.01 ; 0.1 ; 0.2]$ (Methods). While $n=129$ and $n=255$ were in the range of what is recommended for $n$ (Sander et al., 1998), the clustering was worse than for the default parameter $(n=5)$ (Supplementary Table S7). Specifically, the number of outliers exploded for these large neighborhood sizes (Supplementary Table S7, Supplementary Fig. S6).

Since FunFams differ substantially in the number of proteins, we hypothesized that-similar as for $\theta$-it could be reasonable to choose a different $n$ for each FunFam. However, this did not improve compared to the default clustering (Supplementary Table S7); the default $n=5$ was a good choice.

\subsection{Clustering increased purity of ligand binding}

Another way to assess the purity of molecular protein function in protein families is to compare their similarity in terms of ligandbinding. We extracted bound ligands from BioLip (Yang et al., 2013) and only considered annotations defined as the cognate ligand (Tyzack et al., 2018) (SOM_1.3). Of the 13011 FunFams considered so far, $950(7 \%)$ contained any annotation about a ligand bound, and of those 950, $158(17 \%)$ were annotated with more than one unique ligand. Embedding-based clustering split $33 \%$ of these FunFams into clusters with only one type of ligand, i.e., 'pure' clusters (compared to $52 \%$ for EC level 4 ) and an average purity of $36 \%$ (compared to $63 \%$ for ECs). Although ligand annotations remained limited, these results confirmed that embedding-based clustering increased functional purity of FunFams for an aspect of function not used during method development.

\subsection{Increased purity for non-FunFam sequence families} We expected the approach to succeed for any protein family grouping. To establish this, we clustered five superfamilies

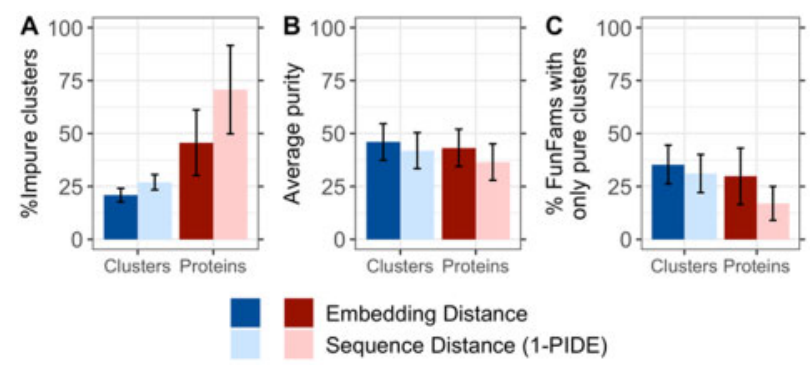

Fig. 5. Clustering results for S35 families. S35 families were created by clustering CATH superfamilies at $35 \%$ sequence identity resulting in 116 impure S35 families. Those were clustered using sequence distance (1-PIDE) and embedding distance. (A) The fraction of impure clusters was higher for the clustering using sequence distance than for the clustering based on embeddings ( $27 \%$ versus $21 \%$ ). (B) Through embedding-based clustering, each impure S35 family was, on average, split into $46 \%$ pure clusters while for the sequence-based clustering, the average purity was $42 \%$. (C) $35 \%$ of impure S35 families were split only into pure clusters for embedding-based clustering and $31 \%$ for sequencebased clustering. Error bars indicate symmetric $95 \%$ confidence intervals. Blue colored bars indicate numbers in terms of clusters, red colored bars in terms of proteins in those clusters. Darker colors indicate values for the clustering while lighter colors indicate values for the random approach.
(Supplementary Table S4) at 35\% PIDE. This resulted in 2160 families-dubbed S35-not optimized for function (SOM_1.4); 116 of the S35 were impure. Embedding-based clustering resulted in $35 \pm 9 \%$ fully pure clusters and an average purity of $46 \pm 9 \%$ (Fig. $5 \mathrm{~B}$ and $\mathrm{C}$, darker blue bars). Clustering by sequence distance (1PIDE) led to only $31 \pm 9 \%$ fully pure clusters and an average purity of $42 \pm 9 \%$ (Fig. $5 \mathrm{~B}$ and C, lighter blue bars). While the difference between sequence- and embedding-based clustering was less than for FunFams, the comparison was very conservative in that the construction of the S35 implicitly favored sequence similarity. Splitting the S35 families into consistent functional clusters worked surprisingly well compared to FunFams because the S35 were less well grouped by function than FunFams, i.e., some functional inconsistency was easy to identify. Given that the construction of the S35 made no other assumption than relation by sequence (PIDE), this experiment provided a rather conservative proof-of-principle that embedding-based clustering is likely to outperform sequence-based clustering for any grouping of proteins.

\section{Conclusion}

FunFams (Das et al., 2016; Sillitoe et al., 2013) provide a high-quality sub-classification of CATH superfamilies into families of functionally related proteins (Sillitoe et al., 2019, 2020). However, some FunFams are impure and $7 \%$ of all FunFams with EC annotations contain at least two different ECs (Supplementary Fig. S1). Here, we introduced a novel approach toward clustering proteins through embeddings derived from the LM ProtBERT (Elnaggar et al., 2020) and further optimized to capture relationships between proteins within one CATH superfamily (called PB-Tucker). Similarity between embeddings can capture information different from what is captured by sequence similarity. In particular, it can reveal new functional relations between proteins (Asgari and Mofrad, 2015; Hamid and Friedberg, 2019; Littmann et al., 2021; Vig et al., 2020; Villegas-Morcillo et al., 2020; Yang et al., 2018). Clustering all FunFams with more than one EC annotation (impure FunFams) using DBSCAN (Ester et al., 1996) reduced the percentage of impure clusters to $22 \%$ (CI [21\%, 23\%]). An impure FunFam was on average split into $63 \%$ pure clusters (CI [60\%: 66\%]) and more than half $(53 \%$, CI $[50-56 \%])$ of all impure FunFams were split into fully pure sub-families (Fig. 2). This corresponded to a four-fold increase over random clustering (Fig. 3B). In terms of number of proteins (rather than number of clusters), the increase was almost ten-fold. Only $4.8 \%$ (CI [4.4-5.2\%]) of the proteins were in FunFams split into pure clusters for random while this number rose to $38 \%$ (CI [33-43\%]) for the PB-Tucker embeddingbased clustering.

A more detailed analysis of five hand-picked superfamilies (Supplementary Table S5) showed that the default choices for the DBSCAN parameters were reasonable (Supplementary Fig. S6, Supplementary Table S7); with the default $n=5$ and the distance threshold $\theta$ determined automatically. Also, Euclidean distance between embeddings worked best (Supplementary Table S7).

Restricting the analysis to manually curated EC annotations limited the validation of our approach to a small fraction $(6.1 \%)$ of all FunFams and even for those FunFams, most EC annotations remain unknown. Nevertheless, we have shown that our approach could capture more fine-grained functional relationships and enabled splitting FunFams into more functionally consistent sub-families. Especially for FunFams without many known functional annotations, our clustering can be used to (i) investigate whether or not the family could be impure based on the number of clusters resulting from the embedding-based split, or (ii) more safely infer functional annotations between members of one functional cluster than between members of one FunFam. We presented evidence suggesting that the findings for EC annotations will hold for other aspects of protein function, e.g. for binding. While we only applied this approach to FunFams using embeddings optimized for $\mathrm{CATH}$, this clustering could be applied to any database of functional families using a more generalized version of those embeddings. 


\section{Acknowledgements}

Thanks to Tim Karl and Inga Weise (both TUM) for invaluable help with technical and administrative aspects of this work. They were happy to acknowledge Ian Sillitoe (UCL) for helpful comments on EC data. Thanks also to the anonymous reviewers who helped substantially to improve the paper. Last, but not least, thanks to all maintainers of public databases and to all experimentalists who enabled this analysis by making their data publicly available.

\section{Funding}

This work was supported by the Bavarian Ministry of Education through funding to the TUM, by a grant from the Alexander von Humboldt foundation through the German Ministry for Research and Education (BMBF: Bundesministerium für Bildung und Forschung), and by two grants from BMBF[031L0168 and Program 'Software Campus 2.0 (TUM)': 01IS17049] as well as by a grant from Deutsche Forschungsgemeinschaft [DFG-GZ: RO1320/4-1]. The authors gratefully acknowledge the support of NVIDIA Corporation with the donation of one Titan GPU used for this research. Nicola Bordin acknowledges financial support from the Biotechnology and Biological Sciences Research Council (UK) [BB/R009597/1].

Conflict of Interest: none declared.

\section{References}

Alley,E.C. et al. (2019) Unified rational protein engineering with sequence-based deep representation learning. Nat. Methods, 16, 1315-1322.

Asgari,E. and Mofrad,M.R. (2015) Continuous distributed representation of biological sequences for deep proteomics and genomics. PLoS One, 10, e0141287.

Bahdanau,D. et al. (2016) Neural machine translation by jointly learning to align and translate. arXiv, 1409.0473.

Becker,S. and Hinton,G.E. (1992) Self-organizing neural network that discovers surfaces in random-dot stereograms. Nature, 355, 161-163.

Beyer,K. et al. (1999) When is "nearest neighbor" meaningful? In: International Conference on Database Theory. Springer, Berlin, Heidelberg. pp. 217-235.

Bromley,J. et al. (1993) Signature verification using a "Siamese" time delay neural network. In Proceedings of the 6th International Conference on Neural Information Processing Systems. Morgan Kaufmann Publishers Inc., Denver, Colorado, pp. 737-744.

Capra,J.A. and Singh,M. (2008) Characterization and prediction of residues determining protein functional specificity. Bioinformatics, 24, 1473-1480.

Dallago,C. et al. (2021) Learned embeddings from deep learning to visualize and predict protein sets. Curr. Protoc. Bioinf., 1, e113.

Das,S. et al. (2015) CATH FunFHMMer web server: protein functional annotations using functional family assignments. Nucleic Acids Res., 43, W148-W153.

Das,S. et al. (2016) Functional classification of CATH superfamilies: a domain-based approach for protein function annotation. Bioinformatics, 32, 2889.

Das,S. et al. (2020) CATH functional families predict protein functional sites. Bioinformatics, 37, 1099-1106.

Devlin,J. et al. (2019) BERT: pre-training of Deep Bidirectional Transformers for Language Understanding. arXiv, 1810.04805. [cs].

Elnaggar,A. et al. (2020) ProtTrans: towards cracking the language of life's code through self-supervised deep learning and high performance computing. bioRxiv.

Ester,M. et al. (1996) A density-based algorithm for discovering clusters in large spatial databases with noise. In: KDD, Portland, Oregon. pp. 226-231.

Hamid,M.N. and Friedberg,I. (2019) Identifying antimicrobial peptides using word embedding with deep recurrent neural networks. Bioinformatics, 35, 2009-2016.

Hannenhalli,S.S. and Russell,R.B. (2000) Analysis and prediction of functional sub-types from protein sequence alignments. J. Mol. Biol., 303, 61-76.

Heinzinger,M. et al. (2019) Modeling aspects of the language of life through transfer-learning protein sequences. BMC Bioinformatics, 20, 723.
Jeffery,C.J. (2003) Moonlighting proteins: old proteins learning new tricks. Trends Genet., 19, 415-417.

Khersonsky,O. and Tawfik,D.S. (2010) Enzyme promiscuity: a mechanistic and evolutionary perspective. Annu. Rev. Biochem., 79, 471-505.

Le-Khac,P.H. et al. (2020) Contrastive representation learning: a framework and review. IEEE Access, 8, 193907-193934.

Lee,D.A. et al. (2010) GeMMA: functional subfamily classification within superfamilies of predicted protein structural domains. Nucleic Acids Res., $38,720-737$.

Littmann,M. et al. (2021) Embeddings from deep learning transfer GO annotations beyond homology. Sci. Rep., 11, 1160.

Liu,J. and Rost,B. (2004) CHOP proteins into structural domain-like fragments. Proteins Struct. Funct. Bioinf., 55, 678-688.

MacDougall,A. et al.; UniProt Consortium. (2020) UniRule: a unified rule resource for automatic annotation in the UniProt Knowledgebase. Bioinformatics, 36, 4643-4648.

Madani,A. et al. (2020) ProGen: language modeling for protein generation. bioRxiv.

Morgat,A. et al.; UniProt Consortium. (2020) Enzyme annotation in UniProtKB using Rhea. Bioinformatics, 36, 1896-1901.,

Orengo,C.A. et al. (1997) CATH-a hierarchic classification of protein domain structures. Structure, 5, 1093-1108.

Pedregosa,F. et al. (2011) Scikit-learn: machine learning in Python. J. Mach. Learn. Res., 12, 2825-2830.

Peters,M.E. et al. (2018) Deep contextualized word representations. arXiv, 1802.05365. [cs]

Radford,A. et al. (2018) Improving language understanding by generative pre-training, 12.

Rao,R. et al. (2019) Evaluating Protein Transfer Learning with TAPE. arXiv, 1906.08230. [cs, q-bio, stat]

Rives,A. et al. (2020) Biological structure and function emerge from scaling unsupervised learning to 250 million protein sequences. bioRxiv.

Sander,J. et al. (1998) Density-based clustering in spatial databases: the algorithm GDBSCAN and its applications. Data Min. Knowledge Discov., 2, 169-194.

Scheibenreif,L. et al. (2019) FunFam protein families improve residue level molecular function prediction. BMC Bioinformatics, 20, 400.

Sillitoe,I. et al. (2020) CATH: increased structural coverage of functional space. Nucleic Acids Res., 49, D266-D273.

Sillitoe,I. et al. (2013) New functional families (FunFams) in CATH to improve the mapping of conserved functional sites to 3D structures. Nucleic Acids Res., 41, D490-498.

Sillitoe,I. et al. (2019) CATH: expanding the horizons of structure-based functional annotations for genome sequences. Nucleic Acids Res., 47, D280-D284.

Steinegger,M. et al. (2019) HH-suite3 for fast remote homology detection and deep protein annotation. BMC Bioinformatics, 20, 473.

The UniProt Consortium. (2019) UniProt: a worldwide hub of protein knowledge. Nucleic Acids Res., 47, D506-D515.

Tyzack,J.D. et al. (2018) Ranking enzyme structures in the PDB by bound ligand similarity to biological substrates. Structure, 26, 565-571.e563.

Vig,J. et al. (2020) BERTology meets biology: interpreting attention in protein language models. arXiv.

Villegas-Morcillo,A. et al. (2020) Unsupervised protein embeddings outperform hand-crafted sequence and structure features at predicting molecular function. Bioinformatics, 37, 162-170.

Webb,E.C. (1992) Enzyme Nomenclature: Recommendations of the Nomenclature Committee of the International Union of Biochemistry and Molecular Biology on the Nomenclature and Classification of Enzymes. Academic Press, San Diego, California.

Weinberger,K.Q. and Saul,L.K. (2009) Distance metric learning for large margin nearest neighbor classification. J. Mach. Learn. Res., 10, 207-244.

Yang,J. et al. (2013) BioLiP: a semi-manually curated database for biologically relevant ligand-protein interactions. Nucleic Acids Res., 41, D1096-D1103.

Yang,K.K. et al. (2018) Learned protein embeddings for machine learning. Bioinformatics, 34, 4138.

Zhou,N. et al. (2019) The CAFA challenge reports improved protein function prediction and new functional annotations for hundreds of genes through experimental screens. Genome Biol., 20, 244. 